\title{
Attracting earlier referrals to a paediatric palliative care service: the vocabulary of enhanced supportive care
}

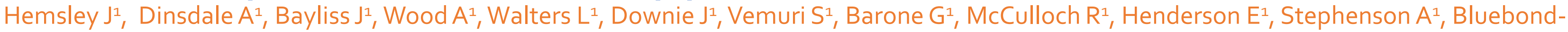
Langner $\mathrm{M}^{1,2}$. Great Ormond Street Hospital ${ }^{1}$ Institute of Child Health ${ }^{2}$

Correspondence: june.hemsley@gosh.nhs.uk

\section{Background}

- Previous studies have shown cancer patients benefit from earlier referral to palliative care services ${ }^{1,2}$.

- Resistance amongst cancer specialists to refer before curative treatment options are exhausted

- An NHS England initiative in adult cancer services aimed to improve early referral by implementing a framework of enhanced supportive care (ESC) 3

- The subject - a tertiary level children's hospital with cancer services and a dedicated specialist palliative care service - agreed to trial this initiative within a paediatric cohort.

\section{Aim and objectives}

Increase rate of early referrals by cancer teams through

- Implementing a shared vocabulary of 'ESC' and 'poor prognosis' (table 1) to facilitate earlier referrals.

- Offering an ESC referral to patients with a poor prognosis within one week of patient and family being informed of prognosis.

\section{Methods}

- The vocabulary of ESC was integrated into the Principle Treatment Centre (PTC)

- An agreed definition of "poor prognosis" was developed in discussions between palliative care and cancer specialists.

Table 1. Definition of poor prognosis

\begin{tabular}{|c|c|c|}
\hline Speciality & Cancer Type & Point of disease trajectory \\
\hline \multirow[t]{2}{*}{ Neuro-oncology } & $\begin{array}{l}\text { DIPG, HGG, HR MBL, ATRT, } \\
\text { ETANTR, metastatic } \\
\text { Ependymoma, Anaplastic } \\
\text { astrocytoma grade III+ }\end{array}$ & At diagnosis \\
\hline & All brain tumours & $\begin{array}{l}1^{\text {st }} \text { relapse / progression / } \\
\text { refractory disease }\end{array}$ \\
\hline \multirow[t]{2}{*}{ Oncology } & Rhabdoid, metastatic RMS & At diagnosis \\
\hline & $\begin{array}{l}\text { RMS, HR HBL, HR NBL (poor } \\
\text { prognosis at day 8o) }\end{array}$ & $\begin{array}{l}1^{\text {st }} \text { relapse / progression / } \\
\text { refractory disease }\end{array}$ \\
\hline \multirow[t]{2}{*}{ Haematology } & Therapy-related AML & At diagnosis \\
\hline & $\begin{array}{l}\text { Burkitt's lymphoma, diffuse } \\
\text { large B cell lymphoma, } \\
\text { haematology post BMT }\end{array}$ & $\begin{array}{l}\text { 1st relapse / progression / } \\
\text { refractory disease }\end{array}$ \\
\hline \multirow[t]{5}{*}{ All specialities } & \multirow[t]{4}{*}{ All malignancies } & $\begin{array}{l}\text { Multiple relapse / progression / } \\
\text { persistent refractory disease } \\
\text { (excl. LCH) }\end{array}$ \\
\hline & & Enrolment in phase $\mathrm{I} / \mathrm{II}$ trial \\
\hline & & $\begin{array}{l}\text { Commencement of } \\
\text { medications for } \\
\text { compassionate use }\end{array}$ \\
\hline & & $\begin{array}{l}\text { Patient's lead } \mathrm{H} / \mathrm{O} \text { consultant } \\
\text { confirms poor prognosis }\end{array}$ \\
\hline & $\begin{array}{l}\text { All malignancies with a } \\
\text { confirmed life-limiting co- } \\
\text { morbidity }\end{array}$ & $\begin{array}{l}\text { Diagnosis of malignancy or } \\
\text { life-limiting co-morbidity } \\
\text { (whichever is later) }\end{array}$ \\
\hline
\end{tabular}

References

1. Wiener et al (2015) Deeper conversations need not wait until the end, JCO, 33 (33) pp 3974;

2. Beecham et al (2016) Keeping all options open: parents' approaches to Advance Care Planning, Health Expectations, DOI: 10.1111/hex.12500 3. https://www.england.nhs.uk/wp-content/uploads/2016/03/ca1-enhncdsupprtv-care-guid.pdf
Defining Enhanced Supportive Care (ESC) in paediatric cancer care

- Symptom management and focus on quality of life

- In parallel with disease-directed treatment

- Poor prognosis or high symptom burden (>grade 2)

Table 2. Symptom assessment criteria

\begin{tabular}{|c|c|c|c|c|c|}
\hline Symptom & Grade o & Grade 1 & Grade 2 & Grade 3 & Grade 4 \\
\hline Pain & Nil pain & Mild pain & $\begin{array}{l}\text { Moderate pain; } \\
\text { limiting instrumental } \\
\text { ADL }\end{array}$ & $\begin{array}{l}\text { Pain at rest; limiting } \\
\text { self-care ADL }\end{array}$ & \\
\hline Peripheral neuropathy & $\begin{array}{l}\text { Nil peripheral } \\
\text { neuropathy }\end{array}$ & $\begin{array}{l}\text { Asymptomatic; loss of } \\
\text { deep tendon reflexes } \\
\text { or paraesthesia } \\
\text { clinical or diagnostic } \\
\text { observations only; } \\
\text { intervention not } \\
\text { indicated }\end{array}$ & $\begin{array}{l}\text { Moderate pain; } \\
\text { limiting instrumental } \\
\text { ADL }\end{array}$ & $\begin{array}{l}\text { Severe symptoms; } \\
\text { limiting self-care } A D L_{i} \\
\text { assistive device } \\
\text { indicated }\end{array}$ & $\begin{array}{l}\text { Life-threatening } \\
\text { consequences; urgent } \\
\text { interventions } \\
\text { indicated }\end{array}$ \\
\hline Nausea & Nil nausea & $\begin{array}{l}\text { Loss of appetite } \\
\text { without alteration in } \\
\text { eating habits }\end{array}$ & $\begin{array}{l}\text { Oral intake decreased } \\
\text { without significant } \\
\text { weight loss, } \\
\text { dehydration or } \\
\text { malnutrition }\end{array}$ & $\begin{array}{l}\text { Inadequate oral } \\
\text { caloric or fluid intake; } \\
\text { tube feeding, TPN or } \\
\text { hospitalization } \\
\text { indicated }\end{array}$ & \\
\hline Vomit & Nil vomiting & $\begin{array}{l}\text { 1-2 episodes } \\
\text { (separated by } 5 \\
\text { minutes) in } 24 \text { hours }\end{array}$ & $\begin{array}{l}\text { 3-5 episodes } \\
\text { (separated by } 5 \\
\text { minutes) in } 24 \text { hours }\end{array}$ & $\begin{array}{l}\text { >=6 episodes } \\
\text { (separated by } 5 \\
\text { minutes in } 24 \text { hours: } \\
\text { tube feeding, TPN or } \\
\text { hospitalization } \\
\text { indicated }\end{array}$ & $\begin{array}{l}\text { Life-threatening } \\
\text { consequences; urgent } \\
\text { interventions } \\
\text { indicated }\end{array}$ \\
\hline Agitation & Nil agitation & Mild mood alteration & $\begin{array}{l}\text { Moderate mood } \\
\text { alteration }\end{array}$ & $\begin{array}{l}\text { Severe agitation; } \\
\text { hospitalization not } \\
\text { indicated }\end{array}$ & $\begin{array}{l}\text { Life-threatening } \\
\text { consequences; urgent } \\
\text { interventions } \\
\text { indicated }\end{array}$ \\
\hline Breathlessness & Nil breathlessness & $\begin{array}{l}\text { Shortness of breath } \\
\text { with moderate } \\
\text { exertion }\end{array}$ & $\begin{array}{l}\text { Shortness of breath } \\
\text { with minimal } \\
\text { exertion; limiting } \\
\text { instrumental ADL }\end{array}$ & $\begin{array}{l}\text { Shortness of breath at } \\
\text { rest; limiting self-care } \\
\text { ADL }\end{array}$ & $\begin{array}{l}\text { Life-threatening } \\
\text { consequences; urgent } \\
\text { interventions } \\
\text { indicated }\end{array}$ \\
\hline Constipation & Bowels open normally & $\begin{array}{l}\text { Occasional or } \\
\text { intermittent } \\
\text { symptoms; occasional } \\
\text { use of stool softeners, } \\
\text { laxatives, dietary } \\
\text { modififation or } \\
\text { enema }\end{array}$ & \begin{tabular}{|l} 
Persistent symptoms \\
with regular use of \\
laxatives or enemas; \\
limiting instrumental \\
ADL
\end{tabular} & $\begin{array}{l}\text { Obstipation with } \\
\text { manual evacuation } \\
\text { indicated limiting } \\
\text { self-care ADL }\end{array}$ & $\begin{array}{l}\text { Life-threatening } \\
\text { consequences; urgent } \\
\text { interventions } \\
\text { indicated }\end{array}$ \\
\hline
\end{tabular}

Results

- Of the 36 patients identified with a new poor prognosis between October 2017 and March 2018, 27 (75\%) were offered an ESC referral within one week.

- Of 6 patients identified with a high symptom burden between January 2019 and March 2019, 5 (83\%) were met within 2 working days; 1 proceeded to palliative care.

\section{Conclusion}

- Attracting earlier referrals to a paediatric palliative care service is feasible through a change of vocabulary and reconfiguration of service.

- Next steps: consider applicability to patients with nonmalignant life-limiting conditions. 